\title{
Assessing the Usage of Dating Sites and Social Networking Sites in Newly Diagnosed HIV Positive Men Who Have Sex with Men (MSM) in Harris County, Texas, 2014
}

\author{
Najmus Abdullah*, Sudipa Biswas, Weilin Zhou, Hafeez Rehman, Salma Khuwaja and \\ Raouf R. Arafat
}

Houston Health Department, City of Houston, Houston, TX, USA

\section{Objective}

To assess the usage of dating sites and social networking sites for finding sexual partners among newly diagnosed HIV positive MSMs in Harris County in 2014

\section{Introduction}

Internet based technologies are becoming quite prominent among today's generation due to its easy accessibility through computer or phone devices [1]. Internet's relative anonymity leads high risk groups to find it easier to meet sexual partners with similar characteristics through dating sites like Grindr, Jack'D, Adams4Adams etc. and mainstream social networking sites like Facebook, Twitter, or Instagram. According to various studies, young MSMs prefer to use dating sites and social networking sites more as a source to meet sexual partners than older MSMs [2].

\section{Methods}

Population-based surveillance data was generated from Houston Enhanced HIV/AIDS reporting system (ehars) for all newly diagnosed HIV positive MSM's in 2014. Data regarding usage of social networking sites as well as dating sites was gathered from the Disease Intervention Specialist (DIS) interviews from Sexually Transmitted Disease Management Information System (STDMIS). Descriptive analysis was performed to identify the distribution of dating and social networking sites usage across different age and race/ ethnicity groups. Logistic regression analysis was used to examine the association of race with the usage of social networking sites and dating sites after adjusting for age. Data was analyzed using Stata Version 13 software.

\section{Results}

The study findings indicate that 207 (32.2\%) out of 643 MSMs used dating sites for finding sexual partners. On the other hand, only $22(3.4 \%)$ out of 643 MSMs used social networking sites. Overall $20(3 \%)$ out of 643 MSMs were not specific about their choice of internet sites for meeting their partners. Among different age groups, young MSMs (18-24 years old) are most likely to prefer dating sites (38\%) compared to mainstream social networking sites (6\%). White MSMs prefer dating sites (33\%) over social networking sites (2\%), same ratio holds true for the African American MSMs preference of dating sites (31\%) vs. social networking sites (2\%) and Hispanic MSMs preference of dating sites (32\%) vs. social networking sites (4\%). MSMs preferences of the dating sites are as follows: Grindr (15.5\%), Adams4Adams (9.49\%), Jack'D (8.71\%), Phone apps like Mocospace/ Badoo (2.95\%), Scruff (2.02\%) and Craigslist (1.87\%). Grindr was favorite choice of finding partners among Hispanic MSM (7.15\%) followed by White MSM (4.04\%) and African American MSM (3.27\%). Facebook (2.8\%) was the only preference among many social networking sites for finding partners. African American MSMs had a significantly lower likelihood of using social networking sites in to White MSMs (OR: 0.61; 95\% CI: 0.37-0.99). Similarly, African American MSM (OR: 0.54; 95\% CI: 0.34-0.87) and Hispanic MSM (OR: $0.51 ; 95 \%$ CI: $0.32-0.82$ ) had significantly lower likelihood of using dating sites compared to White MSM.

\section{Conclusions}

Overall, a trend is seen among MSM who are more comfortable communicating and pursuing sexual partners through the dating sites than social networking sites. Our study shows the dating sites Grindr, Adams4Adams and Jack'D being more popular among Hispanic MSM, and African American MSM than White MSM. White MSM prefers social networking than Hispanics and African Americans may be because of the disparity in socio economic status and accessibility to networking sites. Popular dating sites like Grindr, Adams4Adams, and Jack'D, as well as mainstream social networking sites like Facebook could be used for culturally targeted HIV prevention programs among

at risk populations by community based organizations.

\section{Keywords}

MSM; INTERNET; HIV; DATING SITES; SOCIAL NETWORKING SITES

\section{Acknowledgments}

Houston's HIV Surveillance Program

\section{References}

[1] Holloway IW, Dunlap S, Del pino H, Hermanstyne K, Pulsipher C. Online social networking, sexual risk and protective behaviors: Considerations for clinicians and researchers. NIH Public Access. 2014 Sept; 1 (3):220-228.

[2] Rietmeijer C, Bull S, Mcfarlane M, Patnaik J, Douglas J. Risks and benefits of the internet for populations at risk for sexually transmitted infections (STIs): results of an STI clinic survey. Sexually Transmitted Disease. 2003 Jan; Volume 30 (1):15-19.

\footnotetext{
*Najmus Abdullah

E-mail: Najmus.Abdullah@houstontx.gov
} 